\title{
Custo da Obesidade na Adolescência entre 2008 e 2018 a Partir dos Dados do Datasus
}

\author{
Ana Regina Leão Ibiapina Moura, ${ }^{1}$ Andreia Aparecida Andrade de Santana, ${ }^{2}$ \\ Jaine Francielle Ribeiro de Alencar, ${ }^{2}$ Tuane Rodrigues de Carvalho, ${ }^{2}$ \\ José Fernando Vila Nova de Moraes ${ }^{3}$
}

\begin{abstract}
RESUMO
O objetivo do presente estudo foi analisar os gastos do Sistema Único de Saúde (SUS) com o tratamento da obesidade em adolescentes de 15 a 19 anos. Os dados foram analisados a partir do Serviço de Informação Hospitalares (SIH) do SUS, processados e disponibilizados pelo Departamento de Informática do SUS (Datasus). As opções de pesquisa escolhidas foram baseadas nos gastos com o tratamento da obesidade para a faixa etária de 15 a 19 anos, sexo masculino e feminino e as diferentes Regiões do país. Foram analisados os anos de 2008 a 2018. Percebeu-se um aumento no gasto com o tratamento da obesidade nessa faixa etária. Em 2008 foram gastos $R \$ 190.015,83$, enquanto em 2018 o valor total foi de $R \$ 918.564,40$. O valor total dos gastos no período observado foi de $R \$ 5.570 .080$, 93. Quando analisados por sexo, entre 2008 e 2018 notou-se que as meninas foram mais onerosas do que os meninos ( $R \$ 4.035 .282,05$ vs. $R \$ 1.534 .798,88$, respectivamente). Já na comparação entre as Regiões, fica evidente um maior gasto no tratamento da obesidade na Região Sul, seguida da Região Sudeste. Conclui-se que nos últimos anos houve um aumento substancial com o gasto do tratamento da obesidade em adolescentes, e que isto acompanha o aumento da prevalência de excesso de massa corporal nessa faixa etária. Ademais, as meninas mostraram-se mais caras para o sistema e as Regiões Sul e Sudeste concentraram grande parte destes gastos.
\end{abstract}

Palavras-chave: Obesidade. Custos diretos. Adolescente.

\section{COSTS OF OBESITY IN ADOLESCENCE BETWEEN 2008 AND 2018 ACCORDING TO DATA FROM DATASUS}

\section{ABSTRACT}

The aim of the present study was to analyze the expenses of the Brazilian Public Health System (SUS) with the treatment of obesity in adolescents aged 15 to 19 years. Data were analyzed through the Brazilian Hospital Information System (SIH) of the SUS, which are processed and made available by the Technology Department of the SUS (Datasus). The options used in the research were based on the expenses with the treatment of obesity of adolescents aged 15 to 19 years, of both sexes, and in the different regions of Brazil. Data was analyzed from 2008 to 2018. An increase in the expenses with the treatment of obesity for the cited ages was seen. In 2008, $R \$ 190,015.83$ was spent, while in 2018 this value increased to $R \$ 918,564.40$. The total amount spent in the analyzed period was $R \$ 5.570,080.93$. When analyzed by sex, between 2008 and 2018 , girls cost more to the public system than boys ( $R \$ 4,035,282.05$ vs. $R \$ 1,534,798,88$, respectively). Regarding regions, the South and Southeast Regions were the one that cost more to the Brazilian public health system. It is concluded that there has been a substantial increase in the costs of the treatment of obesity in adolescents, accompanying the increase of excessive weight in this age span. Moreover, girls cost more to the system and the South and Southeast Regions concentrated most of these expenses.

Keywords: Obesity. Direct costs. Adolescent.

\footnotetext{
Instituto Federal de Educação, Ciência e Tecnologia do Maranhão (Ifma) - São Luís/MA, Brasil; Universidade Federal do Vale do São Francisco (Univasf) Petrolina/PE, Brasil.

Universidade Federal do Vale do São Francisco (Univasf) - Petrolina/PE, Brasil.

3 Autor correspondente. Universidade Federal do Vale do São Francisco (Univasf) - Av. José de Sá Maniçoba, s/n - Centro, 56304-205, Petrolina/PE, Brasil. http://lattes.cnpq.br/8490528692298086. https://orcid.org/0000-0002-7394-7700. josefernando.moraes@univasf.edu.br
} 


\section{INTRODUÇÃO}

A obesidade infantil é analisada como um dos mais graves problemas de saúde pública atualmente (BLACK; HUGHES; JONES, 2018), pela sua magnitude e problemas associados, que comprometem a saúde no curto e longo prazos (THE GBD, 2015 OBESITY COLLABORATORS, 2017). Os números são alarmantes, segundo dados da Organização Mundial de Saúde (OMS). Enquanto apenas 1\% das crianças e adolescentes apresentavam obesidade em 1975, em 2016 a obesidade acometia mais de 124 milhões (6\% das meninas e $8 \%$ dos meninos) de crianças e adolescentes.

Este aumento é considerado uma epidemia global, que representa um grande desafio para os sistemas de saúde (WHO, 2016), tanto em termos de suas demandas atuais quanto em projeções de orçamentos futuros de saúde (DOHERTY et al., 2017).

A obesidade infantil é preocupante não só pelo alto risco de persistência na idade adulta, mas também pelo risco elevado de condições de saúde graves durante a infância, como hipertensão arterial, hiperglicemia e dislipidemia, configurando um fator de risco importante para a síndrome metabólica (BLACK; HUGHES; JONES, 2018), consequências adversas à saúde física e mental (LLEWELLYN et al., 2016). Por todas estas consequências, segundo a OMS (WHO, 2016), o excesso de massa corporal e a obesidade está ligado a mais mortes em todo o mundo do que baixo peso.

Em âmbito de sistema público de saúde, o sobrepeso e a obesidade representam um grande fardo econômico (HAYES et al., 2016). Estudos apontam para uma associação entre crianças em idade escolar com sobrepeso e obesidade e maior uso de medicação (SOLMI; MORRIS, 2015), maior número de visitas ambulatoriais e emergência hospitalar (LYNCH et al., 2015) e maior custo com saúde (CLIFFORD et al., 2015) quando comparadas a crianças eutróficas. No Brasil, em 2011, os gastos hospitalares e ambulatoriais do SUS com obesidade chegaram a US\$269,6 milhões, dos quais quase $24 \%$ eram atribuíveis à obesidade mórbida (GONÇALVES; SILVA, 2018).

$\mathrm{O}$ investimento na saúde das crianças e adolescentes é uma das metas do plano de enfrentamento da obesidade infantil proposto pela OMS, o qual prevê metas globais e ações para combater esta epidemia até 2025. Segundo a proposta, investir na saúde das crianças e adolescentes ajudará a atingir as metas globais de saúde e reduzir substancialmente os custos previstos de saúde e econômicos da obesidade (WHO, 2014).
Estudos sobre a relação dos custos com serviços hospitalares gastos com crianças e adolescentes com obesidade, permitem uma melhor compreensão da interação do paciente com o sistema de assistência hospitalar de saúde, e são de interesse no planejamento institucional, desenho de intervenções e de políticas públicas direcionadas (FINKELSTEIN; GRAHAM; MALHOTRA, 2014).

No sistema de saúde brasileiro, definido na Constituição da República Federativa do Brasil de 1988 (BRASIL, 1988), verifica-se que os procedimentos de média e alta complexidade estão entre aqueles que consomem a maior parte dos recursos destinados à saúde (JUNIOR; MENDES, 2015). Tem-se observado que a eficiência no uso destes recursos é um dos aspectos que precisa ser considerado para buscar as melhorias desejadas para a saúde (MAKUTA; O'HARE, 2015). Desta forma, o presente estudo teve como objetivo analisar os valores gastos com o tratamento da obesidade em adolescentes no Brasil.

\section{MÉTODOS}

Este estudo possui natureza descritiva e objetivou analisar os valores gastos pelo SUS com o tratamento da obesidade em adolescentes entre 2008 e 2018, de acordo com os dados disponibilizados pelo Serviço de Informações Hospitalares (SIH) do Sistema Único de Saúde (SUS), em conjunto com as Secretarias Estaduais e Municipais de Saúde, sendo processado pelo Departamento de Informática do SUS (Datasus).

Os procedimentos para a coleta de dados foram previamente descritos em outro estudo (MAZZOCCANTE; MORAES; CAMPBELL, 2012). Brevemente, os dados são acessados por meio do site do Datasus, escolhendo a opção "Informações de Saúde (Tabnet)", disponível no menu "Acesso à Informação". Posteriormente, após acessar o Tabnet, deve-se escolher a opção "Epidemiológicas e Morbidade", em seguida "Morbidade Hospitalar do SUS (SIH/SUS) e escolher a opção "Geral, por local de residência - a partir de 2008". Por fim, na caixa de opções "Abrangência geográfica", deve-se clicar na opção "Brasil por Região e Unidade da Federação".

Para ter acesso aos dados, escolheu-se, na caixa denominada "Linha", a opção "Faixa etária 1". $\mathrm{Na}$ caixa denominada "Coluna" foram escolhidas as opções "Sexo" e "Região", e na caixa "Conteúdo" optou-se pela opção "Valor total". No que se refere aos períodos disponíveis, o presente estudo analisou todos os meses (janeiro a dezembro) de cada ano (2008 a 2018). Por último, na caixa "Seleções disponíveis", a 
única escolha realizada foi na opção "Lista de Morbidades CID-10", na qual apenas a obesidade foi selecionada (MINISTÉRIO DA SAÚDE..., 2020a).

A separação por faixas etárias seguiu as informações geradas pelo aplicativo Tabnet ${ }^{\circledR}$ do Datasus, sendo selecionada a faixa etária de 15 a 19 anos. Isto aconteceu pelo fato de outras faixas etárias possuírem diversas lacunas nos valores declarados ao longo dos anos estudados. A comparação da média de gastos entre 2008 e 2018, de acordo com o sexo, foi realizada por meio do Teste-t de Student, no software SPSS 22.0, com o nível de significância de $p<0,05$. Os gráficos do presente estudo foram elaborados no Microsoft Excel 2013 para Windows ${ }^{\circledR}$.

\section{RESULTADOS}

Analisando os valores gastos pelo SUS com o tratamento da obesidade em adolescentes entre 2008 e 2018, com valores gerais para a faixa etária de 15 a 19 anos (Figura 1), verificou-se que há uma indicação de aumento dos custos com serviços hospitalares ao longo do tempo. Em 2008, por exemplo, foram gastos $\mathrm{R} \$ 190.015,83$ no tratamento da doença, enquanto em 2018 esse valor aumentou para $\mathrm{R} \$$ 918.564,40. Ao todo, considerando os gastos entre 2008 e 2018, o custo do tratamento da obesidade em adolescentes no Brasil foi de $\mathrm{R} \$ 5.570 .080,93$.

Figura 1 - Valores gastos pelo SUS com o tratamento da obesidade em adolescentes entre 2008 e 2018

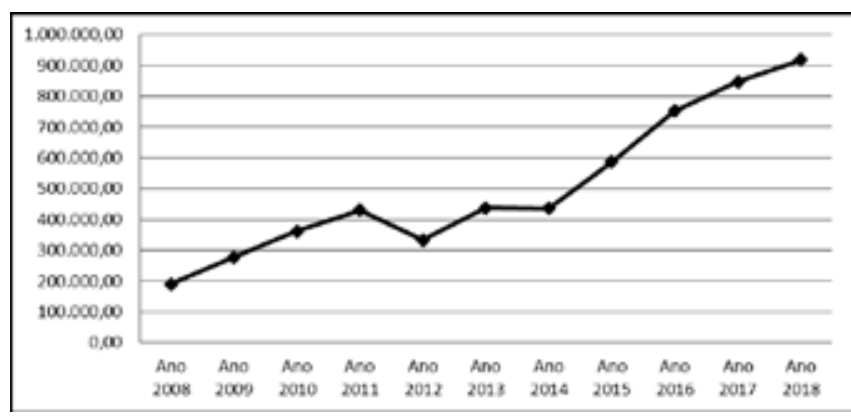

Valores em Reais (R\$).

Fonte: Os autores.

No que se refere aos gastos com o tratamento da obesidade de acordo com o sexo, pode-se identificar, na Figura 2, que os custos mais elevados com a obesidade entre os dez anos analisados foram representados pelo público feminino, que apresentou variações de $R \$ 136.695,33$ (no ano de 2008) para $\mathrm{R} \$ 686.030,84$ (em 2018). Ao todo, foram gastos $\mathrm{R} \$$ 4.035.282,05 no tratamento da obesidade em adolescentes do sexo feminino.
Figura 2 - Valores gastos pelo SUS com o tratamento da obesidade em adolescentes, entre 2008 e 2018, dividido por sexo

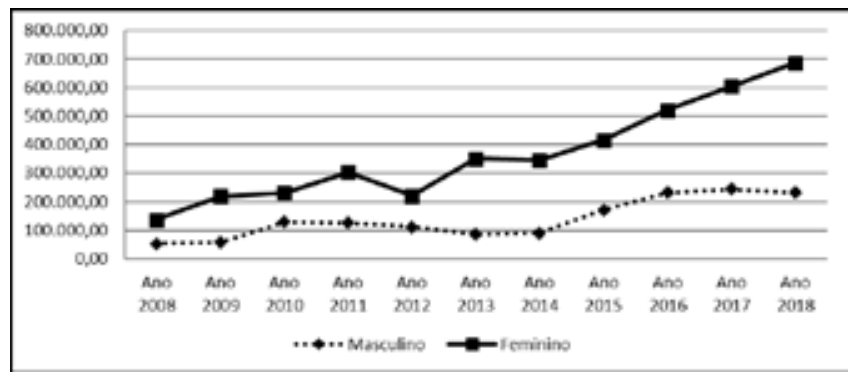

Valores em Reais (R\$).

Fonte: Os autores.

O sexo masculino, por sua vez, apresentou menores gastos, com valores que iniciaram em $\mathrm{R} \$$ $53.320,50$ em 2008 , e subiram para $R \$ 232.533,56 \mathrm{em}$ 2018. Para os adolescentes do sexo masculino, foram gastos, no total, $\mathrm{R} \$ 1.534 .798,88$ entre os anos estudados. Quando comparadas as médias do gasto no período de 2008 a 2018, de acordo com o sexo, foi observada uma diferença estatisticamente significativa, revelando valores maiores para o sexo feminino ( $R \$ 366.834,64 \pm 174.160,08$ vs. $R \$ 139.527,14 \pm$ $70.212,52)\left(t_{(20)}=6,638 ; p=0,018\right)$.

Figura 3 - Custos com a obesidade em todas as regiões do país durante o período de 2008 a 2018,

em adolescentes na faixa etária de 15 a 19 anos

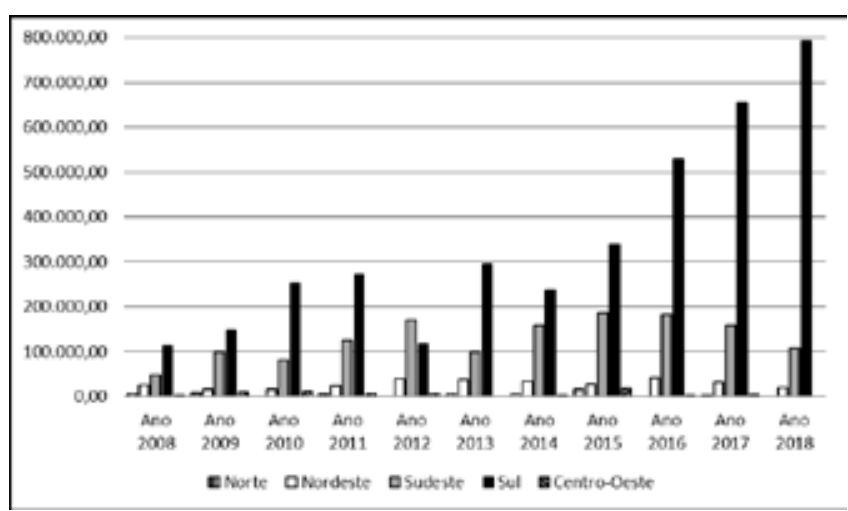

Valores em Reais (R\$).

Fonte: Os autores.

A Figura 3, por sua vez, revela os gastos com o tratamento da obesidade em adolescentes no Brasil de acordo com a Região. Neste cenário, é possível verificar-se que a Região Sul possui o maior registro de gasto, seguido pela Região Sudeste. No ano de 2018, por exemplo, a Região Sul registrou um gasto de R\$ $792.427,36 \mathrm{com}$ o tratamento da obesidade em adolescentes. É válido frisar a ausência de valores para as Regiões em alguns anos, como os anos de 2010, 2012, 2016 e 2018 para a Região Norte, e 2013 e 2018 para a Região Centro-Oeste. 


\section{DISCUSSÃO}

Os resultados do presente estudo apontam para um aumento com gastos com serviços de assistência hospitalar com adolescentes no âmbito do sistema de saúde pública brasileiro. Entre os de 15 a 19 anos, houve aumento dos valores, indicando um maior gasto com adolescentes com obesidade na assistência hospitalar.

Outros estudos têm sugerido uma relação direta entre excesso de massa corporal e internações hospitalares, mesmo em idades precoces. Doherty et al. (2017), por exemplo, indicaram que o excesso de massa corporal aos 13 anos de idade aumenta significativamente a necessidade do uso do sistema de saúde. Já Levitt, Jackson e Morrow (2016), relataram que o custo de uma criança com obesidade aos dez anos de idade, que se mantém obesa na vida adulta, é US\$ $19.000,00$ maior do que uma criança eutrófica, que se mantém com massa corporal normal ao longo da vida.

Os impactos da obesidade nos custos de saúde podem ser justificados pelas diversas comorbidades associadas ao excesso de massa corporal. Segundo dados do Estudo de Riscos Cardiovasculares em Adolescentes (Erica), envolvendo 37.892 adolescentes de 12 a 17 anos de todos os Estados brasileiros, a gravidade da obesidade na adolescência está positivamente associada à elevação dos fatores de risco cardiometabólico, como glicemia, colesterol e triglicerídeos (SBARAINI et al., 2020). Neste contexto, a obesidade aumenta a demanda pela assistência de saúde, o que reflete na oneração dos gastos.

As doenças relacionadas com o excesso de massa corporal, entre os anos de 2008 e 2010, resultaram em um custo direto de $R \$ 2,1$ bilhões para o sistema de saúde pública do Brasil (CODOGNO et al., 2015). Clifford et al. (2015), em seus estudos, evidenciaram uma associação positiva entre obesidade e custos de cuidados de saúde durante a infância. Nesse sentido, para Leach et al. (2020), definir a obesidade como uma doença pode ajudar a garantir melhores fluxos de financiamento para os serviços de tratamento.

Os elevados custos com a obesidade para o sistema de saúde podem envolver questões médicas, psicológicas ou sociais (REINEHR, 2018). Compreender essas questões é importante, pois, além das consequências na adolescência, aqueles que têm obesidade durante esta fase geralmente têm obesidade na idade adulta (REINEHR, 2018). Um estudo na Alemanha (SONNTAG; ALI; DE BOCK, 2016), que quantificou os custos indiretos excessivos durante a vida adulta devido ao sobrepeso e a obesidade durante a infância, ve- rificou um custo excessivo substancial (esperado) durante a vida adulta. $O$ estudo também apontou para a importância do desenvolvimento de intervenções custo-efetivas para prevenção e tratamento da obesidade infantil, assim como alocação de recursos para reduzir a prevalência da obesidade.

Quando comparado entre sexos, os resultados do presente estudo mostraram que os maiores valores são gastos com adolescentes do sexo feminino (valor total de R\$4.095.247,23). Estes resultados corroboram os achados de Hamilton, Dee e Perry (2018), que, ao analisarem os gastos públicos com adolescentes ao longo da vida por meio de uma revisão sistemática, identificaram que meninas têm um gasto maior (€ 26.858 a $€ 30.556$ ) se comparadas com os meninos (17.342 a € 17.692).

Embora os dados apontem maior gasto com o sexo feminino, a Pesquisa Nacional de Saúde do Escolar 2015 (IBGE, 2016) apresentou similaridade entre o excesso de peso e obesidade entre escolares de 13 a 17 anos, dos sexos masculino e feminino. De acordo com o levantamento, $15,4 \%$ dos escolares do sexo masculino apresentaram excesso de massa corporal e $8,3 \%$ foram classificados com obesidade. Para o sexo feminino, por sua vez, a prevalência foi de $16,5 \%$ para excesso de massa corporal e 7,3\% para obesidade. Ainda nesse contexto, Reinehr (2018) menciona que a adesão ao tratamento da obesidade é baixa em adolescentes e a maioria deles, com obesidade, não busca atendimento médico. Assim, acredita-se que o resultado encontrado no presente estudo pode ser um indicativo de diferença na busca por serviços de saúde, falta de registro e/ou indicativo de risco aumentado de consequências da obesidade para o sexo feminino.

Essa hipótese é suportada pelos dados do Sistema de Vigilância Alimentar e Nutricional (Sisvan), do Ministério da Saúde, que permite verificar o quantitativo de atividades relacionadas à organização do processo de trabalho das equipes e as ações de saúde voltadas para a população, realizadas pelos profissionais de saúde da Atenção Básica. De acordo com os relatórios públicos do ano de 2018, 4.531.185 adolescentes do sexo feminino tiveram seu estado nutricional avaliado, enquanto este número foi de apenas 715.612 adolescentes do sexo masculino. De acordo com os relatórios, do total de adolescentes do sexo feminino, $18,91 \%$ apresentou sobrepeso e $8,70 \%$ foram classificados com obesidade. Para o sexo masculino, por sua vez, $16,38 \%$ dos avaliados apresentou sobrepeso e $12,37 \%$ foram classificados com obesidade (MINISTÉRIO DA SAÚDE..., 2020b). 
Ao analisar os resultados por região, observou-se que a Região Sul do Brasil apresentou os maiores gastos com o tratamento de obesidade nos adolescentes. Acredita-se que este achado possa ser justificado por vários fatores, como: inatividade física, comportamento sedentário, padrão alimentar inadequado, álcool e uso de tabaco (SILVA et al., 2016).

Em uma revisão sistemática conduzida por Guimarães Junior et al. (2018), com 27.625 crianças e adolescentes de escolas públicas e particulares em todas as macrorregiões do país, com faixa etária de 5 a 19 anos, foi evidenciado que a prevalência de sobrepeso e obesidade diferem de acordo com a Região. As Regiões que registraram maiores prevalências foram a Sul, com prevalência de sobrepeso e obesidade que variam de $24,6 \%$ a $43,8 \%$, a Sudeste, cuja maior prevalência registrada foi de $41,3 \%$, a Norte, que registrou valores entre $7,4 \%$ a $29,5 \%$, a Centro-Oeste, onde foi encontrado $21,1 \%$ dos meninos e $22,9 \%$ das meninas com sobrepeso, e a Região Nordeste, com a prevalência total de $19,5 \%$ a $30 \%$ de sobrepeso e obesidade, respectivamente. Os relatos da revisão sistemática supracitada corroboram os achados do presente estudo, no sentido de que se espera que a região que apresente maior gasto com o tratamento da obesidade também tenha a maior prevalência de excesso de massa corporal.

Corroborando as informações supra, os dados do Sisvan relacionados ao estado nutricional de adolescentes, do ano de 2018, revelaram que a Região Sul apresentou uma prevalência de $21,36 \%$ para sobrepeso e $14,04 \%$ para obesidade. A Região Sudeste, por sua vez, revelou $19,27 \%$ de sobrepeso e $11,33 \%$ de obesidade. Já para a Região Centro-Oeste, 19,54\% dos adolescentes avaliados apresentaram sobrepeso e $10,45 \%$ de obesidade. Os números da Região Nordeste mostraram $17,70 \%$ de sobrepeso e $7,80 \%$ de obesidade. Por fim, na Região Norte, 17,89\% foram considerados sobrepesados e 6,5\% apresentaram obesidade (MINISTÉRIO DA SAÚDE..., 2020b).

Apesar, todavia, de a Região Sul apresentar uma maior prevalência de sobrepeso e obesidade e os maiores gastos para o tratamento da obesidade, os valores brutos de custeio referentes ao ano de 2018, disponibilizados pelo Fundo Nacional de Saúde (FNS), mostram que não foi a que recebeu maior verba do SUS (MINISTÉRIO DA SAÚDE..., 2020c). A consulta de pagamento consolidada do FNS abrange os valores de custeio referentes a apoio financeiro extraordinário, assistência farmacêutica, atenção básica, atenção de média e alta complexidade ambulatorial e hospitalar, gestão do SUS e vigilância em saúde. De acordo com esses dados, a Região Sudeste foi a que recebeu maior o montante, $\mathrm{R} \$ 30.168 .273 .211,33$, seguida pela Região Nordeste, com $\mathrm{R} \$ 24.284 .678 .755,26$. A Região Sul foi a terceira com maior verba de custeio, totalizando R\$ 11.784.130.024,71. As Regiões Norte e Centro-Oeste apresentaram R\$ 5.895.481.279,86 e R\$ 5.714.896.232,78, respectivamente.

Considerando o cenário discutido, o presente estudo traz informações importantes acerca do crescimento dos gastos do sistema público de saúde no tratamento da obesidade entre a população adolescente no Brasil. Ainda, acentuam-se as disparidades entre os sexos e as diferentes regiões do país. Não é possível descartar, entretanto, a possibilidade de erro de notificação, subnotificação, ou mesmo a adoção de estratégias e políticas locais de enfrentamento da obesidade que possam justificar as disparidades ou ausências de valores apresentados nos diferentes anos avaliados.

\section{CONCLUSÃO}

Conclui-se que os custos totais de saúde para a assistência hospitalar do sistema de saúde público brasileiro são representativos e crescentes no tratamento do excesso de massa corporal e obesidade em adolescentes. Houve disparidades dos valores gastos entre os sexos e nas diferentes Regiões brasileiras. Embora os resultados estejam sujeitos a subnotificações, os mesmos refletem um evento mundial, que é o aumento da obesidade na infância e adolescência. Assim, espera-se que os dados da pesquisa contribuam para o melhor planejamento e gestão dos investimentos e prioridades, auxiliando no processo de eficiência nos gastos em saúde.

\section{REFERÊNCIAS}

BLACK, N.; HUGHES, R.; JONES, A. M. The health care costs of childhood obesity in Australia: An instrumental variables approach. Economics \& Human Biology, New York, v. 31, p. 1-13, Sept. 2018.

BRASIL. Constituição Federal de 1988. Promulgada em 5 de outubro de 1988. Disponível em: http:// http://www.planalto.gov.br/ccivil_03/constituicao/constituicao.htm. Acesso em: 18 set. 2019.

CLIFFORD, S. A. et al. Health-care costs of underweight, overweight and obesity: Australian population-based study. J Paediatr Child Health. Austrália, v. 51, n. 12, p. 1.1991.206, Dec. 2015.

CODOGNO, J. S. et al. The burden of abdominal obesity with physical inactivity on health expenditure in Brazil. Motriz: Revista de Educação Física, Rio Claro, v. 21, n. 1, p. 68-74, jan./mar. 2015. 
DOBBS, Richard et al. Overcoming obesity: an initial economic analysis. New York: McKinsey Global Institute, 2014.

DOHERTY, E. et al. Impact of childhood overweight and obesity on healthcare utilization. Econ. Hum. Biol., New York, v. 27, Part A, p. 84-92, Nov. 2017.

FINKELSTEIN, E. A.; GRAHAM, W. C. K.; MALHOTRA, R. Lifetime direct medical costs of childhood obesity. Pediatrics, Illinois, v. 133, n. 5, p. 854-862, May. 2014.

GONÇALVES, G. M. R.; SILVA, E. N. D. Cost of chronic kidney disease attributable to diabetes from the perspective of the Brazilian Unified Health System. PLoS One, California, v. 13, n. 10, Oct. 2018.

GUIMARÃES JUNIOR, M. S. et al. Fator de risco cardiovascular: a obesidade entre crianças e adolescentes nas macrorregiões brasileiras. Revista Brasileira de Obesidade, Nutrição e Emagrecimento (RBONE), São Paulo, v. 12, n. 69, p. 132-142, jan./fev. 2018.

HAMILTON, D.; DEE, A.; PERRY, J. The lifetime costs of overweight and obesity in childhood and adolescence: a systematic review. Obes. Rev., New Jersey, v. 19, n. 4, p. 452-63, Apr. 2018.

HAYES, A. et al. Early childhood obesity: Association with healthcare expenditure in Australia. Obesity, New Jersey, v. 24, n. 8, p. 1.752-1.758, Aug. 2016.

IBGE. Instituto Brasileiro de Geografia e Estatística. Pesquisa nacional de saúde do escolar: 2015. Coordenação de População e Indicadores Sociais. Rio de Janeiro: IBGE, 2016.

JUNIOR, A. P.; MENDES, A. N. O Fundo Nacional de Saúde e a prioridade da Média e Alta Complexidade. Argumentum, Vitória, v. 7, n. 2, p. 161-177, jul./dez. 2015.

LEACH R. J. et al. Clinical care for obesity: A preliminary survey of sixty-eight countries. Clin. Obes., v. 10, n. 2, p. e12357, 2020.

LEVITT, D. E.; JACKSON, A. W.; MORROW, J. R. An analysis of the medical costs of obesity for fifth graders in California and Texas. International journal of exercise science, Kentucky, v. 9, n. 1, p. 26-33, Jan. 2016.

LLEWELLYN, A. et al. Childhood obesity as a predictor of morbidity in adulthood: a systematic review and meta-analysis. Obes. Rev., New Jersey, v. 17, n. 1, p. 56-67, Jan. 2016.

LYNCH, B. A. et al. Health care utilization by body mass index in a pediatric population. Acad. Pediatr., New York, v. 15, n. 6, p. 644-650, Nov./Dec. 2015.

MAKUTA, I.; O'HARE, B. Quality of governance, public spending on health and health status in Sub Saharan Africa: a panel data regression analysis. BMC Public Health, New York, v. 15, n. 1, p. 932-942, Sept. 2015.

MAZZOCCANTE, R. P.; MORAES, J. F. V. N.; CAMPBELL, C. S. G. Gastos públicos diretos com a obesidade e doenças associadas no Brasil. Revista de Ciências Médicas, Campinas, v. 21, n. 1/6, p. 25-34, Jan./Dez. 2012.
MINISTÉRIO DA SAÚDE. Departamento de Informática do SUS (Datasus). Morbidade hospitalar do SUS (SIH/SUS). Disponível em: https://datasus.saude.gov.br/acesso-a-informacao/morbidade-hospitalar-do-sus-sih-sus/. Acesso em: 4 ago. 2020a.

MINISTÉRIO DA SAÚDE. Fundo Nacional de Saúde (FNS). Consulta de pagamento consolidada. Disponível em: https://consultafns.saude.gov.br/\#/consolidada. Acesso em: 4 ago. 2020c.

MINISTÉRIO DA SAÚDE. Sistema de Vigilância Alimentar e Nutricional (Sisvan). Relatórios consolidados de acesso público. Disponível em: https://sisaps.saude.gov.br/sisvan/relatoriopublico/index. Acesso em: 4 ago. 2020b.

REINEHR, T. Long-term effects of adolescent obesity: time to act. Nature Reviews Endocrinology, New York, v. 14, n. 3, p. 183-188, Nov. 2018.

SBARAINI, M. et al. Severity of obesity is associated with worse cardiometabolic risk profile in adolescents: findings from a Brazilian national study (ERICA). Nutrition, v. 75-76, p. 110758, Jul./Aug. 2020.

SILVA D. R. et al. Association between risk behaviors and adiposity indicators in adolescents from Southern Brazil: A methodological approach. J Child Health Care, v. 20, n. 3, p. 314-323, Aug. 2016.

SOLMI, F.; MORRIS, S. Association between childhood obesity and use of regular medications in the UK: longitudinal cohort study of children aged 5-11 years. BMJ Open. London, v. 5, n. 6, e007373, May. 2015.

SONNTAG, D.; ALI, S.; DE BOCK, F. Lifetime Indirect Cost of Childhood Overweight and Obesity: A Decision Analytic Model. Obesity, New Jersey, v. 24, n. 1, p. 200-206, Jan. 2016.

THE GBD 2015 OBESITY COLLABORATORS. Health effects of overweight and obesity in 195 countries over 25 years. $N$ Engl J Med., Massachusetts, v. 377, n. 1, p. 13-27, Jul. 2017. WHO. World Health Organization. Global nutrition targets 2025: childhood overweight policy brief (WHO/NMH/ NHD/14.6). Geneva: World Health Organization, 2014.

WHO. World Health Organization. Report of the Commission on Ending Childhood Obesity. Geneva: World Health Organization, 2016. 